\title{
Uvular Necrosis as an Unusual Complication of Bronchoscopy Via the Nasal Approach
}

\author{
Lily Kristine F Sunio MD, Tahmeed A Contractor MD, and Gina Chacon MD
}

\begin{abstract}
Bronchoscopy can cause post-procedural throat discomfort due to oropharyngeal irritation. We report a rare complication of bronchoscopy that resulted in similar symptoms. A 30-year-old man complained of severe throat discomfort a day after bronchoscopy. Examination revealed a swollen, elongated uvula. The lower half of the uvula was completely white, consistent with uvular necrosis. He was managed conservatively with topical lidocaine and antihistamines. At a follow-up examination 2 weeks later his symptoms had substantially improved and the necrosed uvula had separated, leaving a shortened uvula with an irregular border. Simple precautions taken during bronchoscopy can avoid this rare complication that causes persistent symptoms. Key words: uvula; necrosis; bronchoscopy; complication; throat discomfort; rare. [Respir Care 2011;56(5):695-697. ( 2011 Daedalus Enterprises]
\end{abstract}

\section{Introduction}

Throat discomfort after orotracheal or nasotracheal procedures is relatively common, occurring in about $40 \%$ of cases, ${ }^{1,2}$ usually due to pharyngeal irritation, and is generally self-limiting. Severe or non-resolving throat discomfort should raise one's suspicion for unusual complications such as uvular necrosis. Though this has been sporadically described after endoscopy, intubation, and vigorous oral suctioning, ${ }^{1-6}$ it has not been reported as a complication of bronchoscopy. We describe a unique case of a 30-year-old man who developed uvular necrosis after bronchoscopy. Simple precautions can easily avoid postbronchoscopy prolonged throat discomfort.

\section{Case Report}

A 30-year-old African-American man presented with a 6-week history of productive cough associated with fever,

Lily Kristine F Sunio MD, Tahmeed A Contractor MD, and Gina Chacon MD are affiliated with the Division of Internal Medicine, Michigan State University, East Lansing, Michigan.

The authors have disclosed no conflicts of interest.

Correspondence: Lily Kristine F Sunio MD, Division of Internal Medicine, B301 Clinical Center, Michigan State University, East Lansing MI 48824-1313. E-mail: lilykristine.sunio@hc.msu.edu.

DOI: $10.4187 /$ respcare.00936 night sweats, and decreased appetite. He was found to have a right-sided cavitary lesion on chest radiograph, which was confirmed on a contrast-enhanced computerized tomogram. After starting broad-spectrum intravenous antibiotics, we evaluated further with diagnostic bronchoscopy.

During the bronchoscopy, topical anesthesia was provided with $10 \%$ lidocaine spray to the oropharynx and $2 \%$ lidocaine jelly to the nostrils. Supplemental oxygen at $6 \mathrm{~L} / \mathrm{min}$ was given through the left nostril. He was sedated with intravenous fentanyl and midazolam. With the patient in the supine position, we introduced a plain bronchoscope (BF-160, Olympus, Center Valley, Pennsylvania) through the right nostril and advanced into the tracheobronchial tree. Oral suctioning with a Yankauer suction device was performed intermittently during bronchoscopy. After confirming that there were no endobronchial lesions, the right upper lobe was lavaged with $20-\mathrm{mL}$ aliquots of saline, which was collected in a specimen trap and submitted for cytology and culture. He tolerated the approximately 30 -min procedure well.

On the following day he complained of an intense foreign-body sensation in the throat, and odynophagia. Oropharyngeal examination revealed a swollen, erythematous, and unusually elongated uvula, the lower half of which measured $1 \times 2 \mathrm{~cm}^{2}$, transformed into whitish tissue. There was a clear line of demarcation at the mid-uvula (Fig. 1). The remainder of his oropharynx, including the palate, tongue, and tonsils, was unremarkable. The clinical 


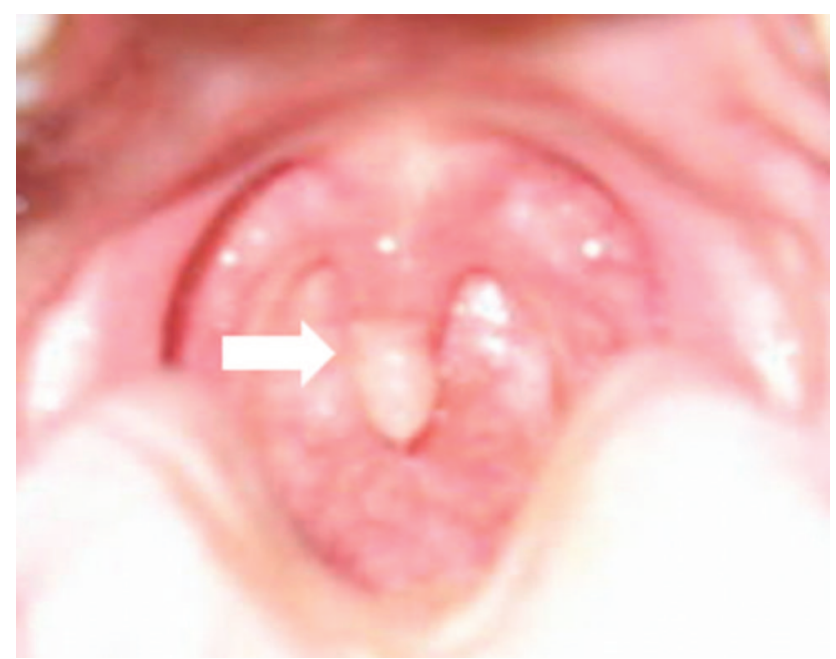

Fig. 1. The whitish-colored lower half of the uvula (arrow) is necrotic one day after bronchoscopy.

findings were consistent with uvular necrosis. He was reassured and managed symptomatically with topical lidocaine and antihistamines.

On day 4 of his hospital stay, his cough and fever had substantially improved. An extensive infectious disease workup, including blood and bronchial-wash cultures for bacteria, mycobacteria, and fungi, was negative. Given his clinical response to antibiotics, bacterial infection was presumed to cause the lung pathology. He was discharged on clindamycin, and a workup for connective-tissue diseases (such as Wegener's granulomatosis) was deferred.

On a follow-up visit 2 weeks later, his oropharyngeal symptoms had resolved. The necrotic uvular tissue had detached from the rest of the uvula, leaving an irregular lower uvular border (Fig. 2). At the 4th-week follow-up he reported no recurrence of oropharyngeal symptoms, fever, or night sweats, and chest radiograph revealed complete resolution of the cavitary lesion, with minimal scarring.

\section{Discussion}

Sore throat can occur after bronchoscopy and other procedures that involve manipulation of the pharyngeal area, due to a wide variety of causes (Table 1). Our patient had an unusual cause of sore throat after bronchoscopy. Though uvular necrosis has been described after intubation, endoscopy, and vigorous, blind suctioning, ${ }^{1-6}$ it has not been previously reported following bronchoscopy.

Patients with elongated uvula, such as our patient, are predisposed to uvular necrosis. Compression of the uvula against surrounding structures and the resultant impaired blood flow lead to necrosis. ${ }^{1,2}$ Since our patient was in the supine position during the procedure, the uvula was probably compressed between the bronchoscope and the pos-

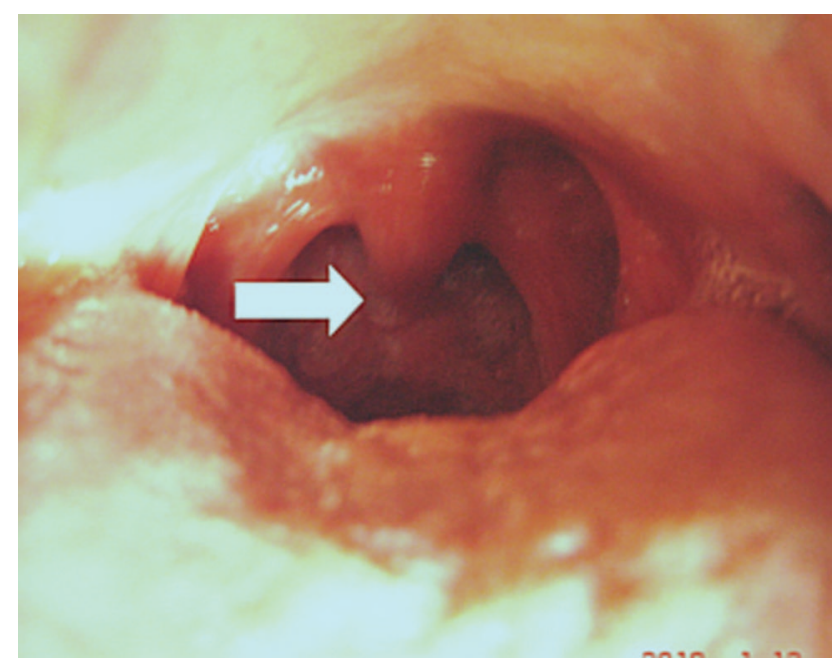

Fig. 2. Two weeks after bronchoscopy the necrotic portion of the uvula had detached from the upper half of the uvula (arrow).

terior pharyngeal wall, which probably compromised vascular supply from the lesser palatine artery and the surrounding vasculature. Suctioning might also have contributed to the injury.

Persistent sore throat is the usual symptom accompanying uvular necrosis, and the diagnosis is clinical. Symptoms typically persist for 2 weeks, with complete resolution after the necrosed part is severed. Management is mostly symptomatic, and these patients have been treated with steroids, antihistamines, and topical epinephrine..$^{1,2,6}$ Calikapan et al recommended excision of the necrotic portion of the uvula. ${ }^{6}$ We gave our patient topical lidocaine and antihistamines, which adequately managed his sore throat, foreign-body sensation, and odynophagia.

It is important to be aware of uvular necrosis as a rare possibility, especially in a patient with an elongated uvula. Vigorous, blind suctioning should be avoided in such patients. Nasopharyngeal bronchoscopy should preferably be

Table 1. Causes and Sites of Post-Procedural Sore Throat 
done in the sitting position. If the oropharyngeal route is used for bronchoscopy, intubation, or endoscopy, the tube or scope should be placed to one side of the midline. These simple precautions can prevent uvular damage.

\section{REFERENCES}

1. Atkinson CJ, Rangasami J. Uvula necrosis: an unusual cause of severe post-operative sore throat. Br J Anesthesia 2006;97(3):426-427.

2. Evans DP, Lo BM. Uvular necrosis after orotracheal intubation. Am J Emerg Med 2009;27(5):631.e3-e4.

3. Gupte AR, Draganov PV. Post-endoscopic retrograde cholagiopancreatography uvular necrosis. Clin Gastroenterol Hepatol 2009;7(10): A18.

4. Tang SJ, Kanwal F, Gralnek IM. Uvular necrosis after upper endoscopy: a case report and review of literature. Endoscopy 2002;34(7): 585-587.
5. Shores NJ, Bloomfield RS. Images in clinical medicine: uvular necrosis after endoscopy. N Engl J Med 2009;361(12):e20.

6. Calikapan GT, Karakus F. Uvula necrosis after endotracheal intubation for rhinoplasty (letter). Aesthetic Plast Surg 2008;32(4):710711.

7. Hilding AC. Laryngotracheal damage during intratracheal anesthesia. Ann Otol Rhinol Laryngol 1971;80(4):565-581.

8. Peppard SB, Dickens JH. Laryngeal injury following short term intubation. Ann Otol Rhinol Laryngol 1983;92 (4 Pt 1):327-330.

9. McHardy FE, Chung F. Postoperative sore throat: cause, prevention and treatment. Anaesthesia 1999;54(5):444-453.

10. Donnelly WA, Grossman AA, Grem FM. Local sequelae of endotracheal anesthesia as observed by examination of one hundred patients. Anesthesiology 1948;9(5):490-497.

11. Jackson C. Contact ulcer granuloma and other laryngeal complications of endotracheal anesthesia. Anesthesiology 1953;14(5): 425-436. 